\title{
Coherent optical effects in two-dimensional nanostructures with semiconductor quantum dots
}

\author{
Alexei Prokhorov ${ }^{1, *}$ and Valentyn Volkov $^{2}$ \\ ${ }^{1}$ Vladimir State University named after A. G. and N. G. Stoletovs, 600000 Vladimir, Russia \\ ${ }^{2}$ Moscow Institute of Physics and Technology, 141700 Dolgoprudny, Moscow region, Russia
}

\begin{abstract}
The effects of quantum coherence arising in an ensemble of semiconductor quantum dots located near the surface of two-dimensional optical materials are considered. The conditions for the realization of strong coupling between surface plasmon-polaritons and quantum dot in proximity to graphene are studied.
\end{abstract}

The realization of strong coupling between the emitter and electromagnetic field in subwavelength systems is one of the priorities in modern nanoplasmonics [1]. Nanosystems supporting strong emitter-field coupling are the basis for the creation of highly efficient surface plasmon-polariton (SPP) sources [1], nano-antennas [2], and sensors [3]. The greatest perspectives are associated with the implementation of strong coupling between the semiconductor quantum dot (QD) and SPP in graphene [4]. The complexification of the graphene geometry allows increasing the density of states for the self-consistent field of emitter near the spatial limiters, e.g., grooves, pyramids, and so forth [5]. The use of QD as an emitter has an advantage over atoms, namely, the possibility to tune the wavelength for obtaining the optimal SPP propagation length for various conductor- dielectric interfaces.

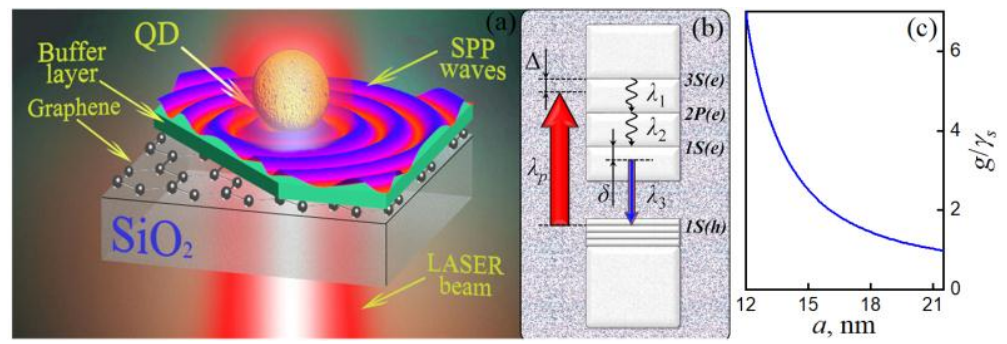

Fig. 1. (a) The scheme of a possible experiment for SPPs generation. (b) The relative position between energy gaps and band offsets of InSb bulk semiconductor with depicted energy levels of QD;

$\lambda_{3}=5.24 \mu \mathrm{m}, \lambda_{p}=1.52 \mu \mathrm{m}$. (c) Dependence of $g / \gamma_{s}$ ratio as a function of the radius of InSb QD placed on $d z_{z b}=2 \mathrm{~nm}$ buffer layer of $\mathrm{SiO}_{2}$ deposited on graphene.

\footnotetext{
*Corresponding author: avprokhorov33@mail.ru
} 
In this work, we investigate the features of the light-to-SPP conversion using QD placed in proximity to graphene. The efficiency of this conversion depends on the type of SPP-QD coupling. The key point in identifying the type of coupling is the ratio between the scattering rate of electrons in graphene $\gamma_{s}$, the effective QD relaxation rate $\Gamma_{\text {eff }}=k_{R} \Gamma_{0}$, and SPP-QD coupling constant $g$. In particular, the inequality $g>\gamma_{s}, \Gamma_{\text {eff }}$ characterizes the regime of strong SPP-QD coupling [4]. We assume that QD is placed on a buffer layer deposited on graphene surface that is located on $\mathrm{SiO}_{2}$ substrate, see Fig. 1a. We propose to implement combined light-to-SPP ladder-type scheme of interaction in QD with infrared $\lambda_{p}$ laser pump and $\lambda_{3}$ signal SPP mode, see Fig. 1a,b. The implementation of ladder interaction scheme in QD (see Fig. 1b) allows for realizing the strong SPP-QD coupling even for the flat graphene. The advantage of the scheme is the ability to tune the pump wavelength far from the SPP wavelength, which solves the problem of background illumination when observing SPP. The coupling constant can be determined as $g(\bar{r})=\sqrt{\omega_{0} / \hbar \varepsilon_{0} V_{E F}} \aleph(\bar{r}) \mu_{12}$, where $\mu_{12}$ is the dipole moment of SPP-induced transition; $\aleph(\bar{r})=E_{\text {eff }}(\bar{r}) / E_{\text {eff }}^{(\max )}$ is the field attenuation at the QD position relative to its maximum value $E_{\mathrm{eff}}^{(\max )}$, which we extract from full-wave simulation; $V_{E F}=\left(\lambda_{3} / n_{E F}\right)^{3}$ is the volume of interaction, $E_{\text {eff }}(\bar{r})$ is the field distribution. The parameter $k_{R}=\left(\Gamma-\Gamma_{S P P}\right) / \Gamma_{0}$ describes the change in relaxation rate [6] of QD in proximity to graphene, where $\Gamma=\Gamma_{0}+9 \Gamma_{0} /\left(2 k_{0}^{3}\right) \int_{0}^{\infty} K\left(k_{\|}\right) \mathrm{d} k_{\|}$is the total relaxation rate, $\Gamma_{S P P}=9 \Gamma_{0} /\left(2 k_{0}^{3}\right) \int_{k_{S P P}-\Delta k}^{k_{S P P}+\Delta k} K\left(k_{\|}\right) \mathrm{d} k_{\|}$is the rate of SPP generation; $k_{0}=2 \pi / \lambda_{0}, k_{\|}$is the in-plane field wave vector. The integrand $K\left(k_{\|}\right)=k_{\|}^{2} \operatorname{Im}\left[-1 /\left(\varepsilon_{d}+1+i k_{\|} \sigma /\left(\varepsilon_{0} \omega\right)\right)\right] e^{-2 k_{\|} z}[4,6]$ depends on the distance between the QD and grapheneand the QD radius. At a fixed distance from QD to the surface, the ratio $g / \gamma_{s}$ increases with decreasing the QD size, which is caused by enhancing the dipole moment of transition $\mu_{12}$ for QD with the small radius, see Fig. 1c. At the same time, the graphene with small values of chemical potential $\mu=0.161 \mathrm{eV}$ demonstrates not so good SPP-QD coupling, which is associated with the short spatial scale of generated SPPs $\left(n_{E F}=440\right)$ and a small area of SPP-QD overlapping. In fact, the highlylocalized field of SPP quickly weakens at the scale of approximately $10 \mathrm{~nm}$ over the graphene surface and "does not feel" the internal QD degrees of freedom. On the other hand, SPPs are less-well localized on doped graphene with a bigger $\mu$, but they propagate over long distance and well overlap the region of QD location. As a result, the ratio $g / \gamma_{s}=1.14$ for $20 \mathrm{~nm} \mathrm{InSb}$ QD placed at $22 \mathrm{~nm}$ distance to graphene with $\mu=0.3 \mathrm{eV}$ demonstrates the possibility for the creation of "bright" SPP source with infrared pumping.

\section{References}

1. S.I. Bozhevolnyi, L. Martin-Moreno, F. Garcia-Vidal, Quantum Plasmonics (Springer Series in Solid-State Sciences), 2017

2. A.E. Krasnok, A.P. Slobozhanyuk, C.R. Simovski et. al., Sci. Rep. 5, 12956 (2015)

3. Y.V. Stebunov, O.A. Aftenieva, A.V. Arsenin, V.S. Volkov, ACS Appl. Mater. Interfaces 7, 21727 (2015)

4. F.H.L. Koppens, D.E. Chang, F.J.G. De Abajo, Nano Lett. 11, 3370 (2011)

5. A.N. Grigorenko, M. Polini, K.S. Novoselov, Nat. Photonics 6, 749 (2012)

6. L. Novotny, B. Hecht, Principles of nano-optics (Cambridge University Press, 2006) 